\title{
Performance Analysis and Comparison of three Kinds of Electrode Structure for Micro-atomizer
}

\author{
Honghua Liao, Binbin Zhou, Man Liu, Yi Lv and Hailing Yuan* \\ School of Information and Engineering, Hubei University for Nationalities, Enshi, China, 445000 \\ ${ }^{*}$ Corresponding author
}

\begin{abstract}
In order to develop the flat-plate micro-atomizer, the flat-plate micro-atomizer based on Ansys software was simulated. In this paper, the primary research work is to discuss three types of electrode configurations. It includes plate-plate, multineedle-plate and screen mesh. They have effect on the characteristic of flat-plate micro-atomizer. Three types of electrode parameters have effect on the characteristic of flat-plate micro-atomizer, such as the electrode thickness, steel needle diameter, distribution distance of steel needle, length of steel needle, screen mesh size and other parametersit. The simulation results show that the discharge density unit would be easy to obtain by many factors, such as the smaller electrode thickness, the smaller steel needle diameter, the smaller distribution of steel needle and the closer mesh. The longer the length of the needle is, the larger current density of the discharge have. Under the same condition, comparison of three types of electrode structure can be know that the current density covered on the screen mesh's electrode is much larger than the plate-plate and multineedle-plate.
\end{abstract}

Keywords-micro-atomizer; dielectric barrier discharge; ansys simulation; ectrode structure; density of discharge

\section{INTRODUCTION}

Atomic spectrometry has been widely used in the analysis and determination of trace elements, which has the ability of detect the wide linear range and multi elements simultaneously. In order to solve the difficulties in the field of analys, it is hoped that a high automation and portable analysis system can continue to emerge ${ }^{[1]}$. In the research work for a variety of micro plasma generating system, micro plasma was used as source of excitation, which has the advantages of small volume, low power consumption, easy fabrication process. If the excitation light source is used to replace the traditional excitation light source, it can give a good prospect for the development of miniaturization of emission spectrum system ${ }^{[2]}$.

Dielectric barrier discharge (DBD) was regard as a microplasma occurrence mode. It has some characteristics, such as simple structure, small volume, low power consumption, low operation temperature, spontaneous and low temperature plasma. DBD can produce the required low temperature plasma under high pressure atmospheric pressure, so it can save the vacuum device $^{[3-4]}$. This technology has been widely used in many fields, such as ozone synthesis, tail gas treatment, polymer surface modification, thin film growth and so on ${ }^{[5]}$. It can produce a large number of free radicals or ions at time of high energy electrons collision with the surrounding gas molecules. The characteristics are determined that the analysis also has a good prospect in the field of science.

The core component of the atomizer structure is the DBD generator, the parameters optimization based on the structure of generator electrode will greatly enhance its function. Therefore, it is an important precondition for the optimization design and improvement of the atomization device to study the discharge density of the electrode structure. It is also the basis for comprehensive analysis of the function of the atomizer ${ }^{[6-8]}$. In this paper, three structures of flat micro-atomizer electrode are analyzed by different kinds of discharge density. It can obtain the optimum design optimization parameters for the flat-plate micro-atomizer design of on-micro-fluidic chip-atomic fluorescence system.

\section{Plate Dielectric Barrier Discharge Electrode MODEL AND EQUIVALENT CIRCUIT}

The micro atomization device is composed of the cavity, the intermediate dielectric layer and the excitation electrode (high voltage electrode and grounding electrode). The forms of multineedle-plate and screen mesh electrode are outside the surfuce of dielectric plate. They are used as high voltage electrode. The high-voltage electrode and the grounding electrode dielectric barrier are located in the center of the dielectric plate. They are the upper and lower symmetry ${ }^{[9-10]}$.

Figure 1 is the DBD equivalent circui. DBD is equivalent to dielectric barrier capacitance $\mathrm{Cd}$ and air gap equivalent capacitance $\mathrm{Cg}$ in series before air gap breakdown. After the air gap breakdown, the switch in the diagram is closed. $\mathrm{Cg}$ and $\mathrm{R}(\mathrm{t})$ of time varying plasma are connected in parallel.

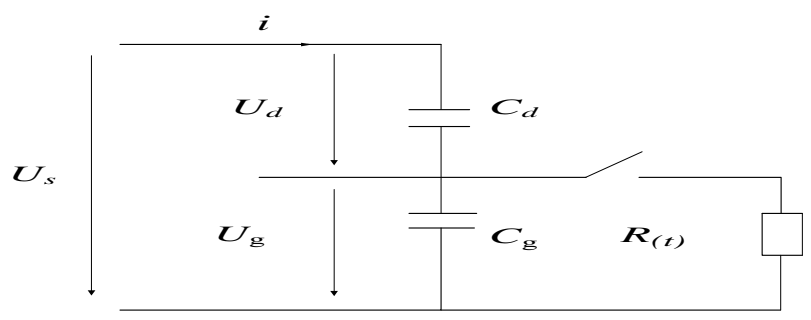

FIGURE I. DBD EQUIVALENT CIRCUIT

\section{DBD MOdELING BASED ON ANSYS}

When using the Ansys software simulation, medium material of the three are all choose as glass, such as the dielectric 
constant of $4.1 \mathrm{~F} / \mathrm{m}$. Their shapes of square are all $53 \times 53 \mathrm{~mm}$. The $33 \times 33 \mathrm{~mm}$ square copper are all choose as the plate electrode. The upper and lower electrode surface is covered by the dielectric. Atomization device electrodes of structure simulation parameters are shown in table. The main structure of Atomizer electrode is simulated. The parameters are shown in the Table 1:

TABLE I. ATOMIZER ELECTRODE MAIN STRUCTURE SIMULATION PARAMETERS

\begin{tabular}{|c|c|c|}
\hline Plate type & $\begin{array}{c}\text { Electrode structure } \\
\text { Parameter }\end{array}$ & Parameter range \\
\hline plate-plate & Electrode thickness $T$ & $1 \sim 3 \mathrm{~mm}$ \\
\hline \multirow{2}{*}{ multineedle-plate } & Needle diameter $\Phi$ & $0.2 \sim 1 \mathrm{~mm}$ \\
\cline { 2 - 3 } & Needle spacing D & $2 \sim 8 \mathrm{~mm}$ \\
\hline the screen mesh & Mesh size $\mathrm{H}$ & $0.3 \sim 0.6 \mathrm{~mm}$ \\
\hline
\end{tabular}

IV. Electrode Discharge Density ANALysis OF Key STRUCTURE PARAMETERS OF ELECTRODES

In order to optimize the design of electrode geometrical parameters, we should first understand the geometric parameters of electrode structure have effect on the performance of the micro-atomizer. According to the structure of atomizer electrode and range of parameter, we choosed the geometric parameters most effected on the atomizer performance for analysis. These parameters include the electrode thickness $\mathrm{T}$, the needle diameter $\Phi$, the needle length $\mathrm{L}$, the distribution distance $\mathrm{D}$ of the needle and the screen mesh size H.They are shown in Figure 2. In this paper, copper electrode was used. The thickness of glass was set to $1 \mathrm{~mm}$. The gap between the plate and board discharge was set to $2 \mathrm{~mm}$. The excitation voltage between the upper and lower electrodes is $10 \mathrm{kV}$. The structure of plate-plate, multineedle-plate and screen mesh are shown in Figure 2(a), (b), (c), respectively.

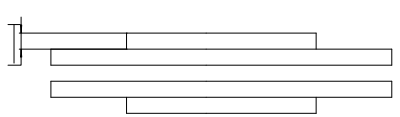

(a)

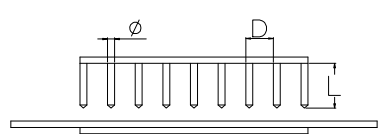

(b)

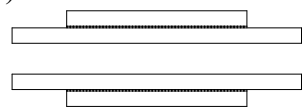

(c)

FIGURE II. THE KEY STRUCTURAL PARAMETERS OF VARIOUS ELECTRODES

\section{A. Effect of Electrode Thickness on Discharge Characteristics}

The electrode thickness $\mathrm{T}$ is the most basic parameters in the electrode structure. The impact of the atomizer is also obvious. In this paper, the electrode thickness is chosen as $1 \sim 3 \mathrm{~mm}$. The electrode is choosen the copper as the discharge material. When the thickness of the copper was changed separately, the effect of different electrode thickness on the discharge characteristics was shown in Figure 3. The thickness of the copper electrode ware $1 \mathrm{~mm}, 2 \mathrm{~mm}, 3 \mathrm{~mm}$, respectively.

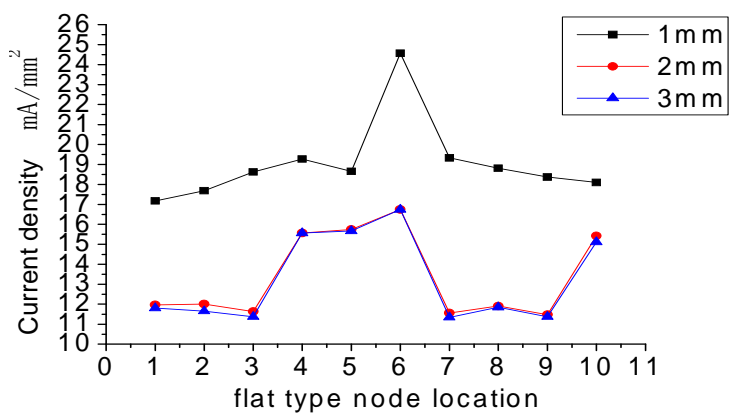

FIGURE III. EFFECTS OF DIFFERENT ELECTRODE THICKNESS ON DISCHARGE CHARACTERISTICS

Figure 3 shows the current density surfaced on different thickness electrodes curves have the same trend. When the electrode thickness are $2,3 \mathrm{~mm}$, the thickness $2 \mathrm{~mm}$ of the current density is slightly larger than the thickness of $3 \mathrm{~mm}$ on the surface of electrode. However, thickness $1 \mathrm{~mm}$ of the current density is largest than others on the surface of electrode. Compared with the curve of Figure 3, under the same condition, the thinner the thickness of the electrode is, the greater the unit current density on the surface of the electrode is.

\section{B. Effect of Steel Needles Diameter on Discharge Characteristics}

The needle diameter $\Phi$ is an important parameter of the multineedle-plate electrode. The effect on the multi-pin-plate electrode is also obvious. In this paper, the range of steel needle diameter is $0.2 \sim 1 \mathrm{~mm}$. The AISI 316 stainless steel is used for the steel needle material. The diameters of the needle is changed individually. The diameters of the pins are $0.2 \mathrm{~mm}, 0.6 \mathrm{~mm}, 1 \mathrm{~mm}$, respectively. The effect of steel needles diameters on the discharge characteristics are shown in Figure 4.

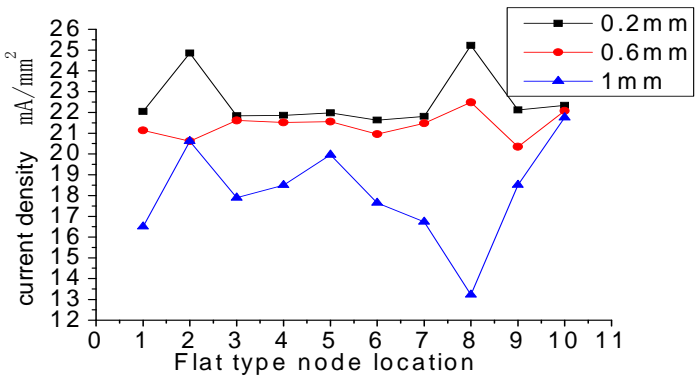

FIGURE IV. EFFECT OF DIFFERENT NEEDLE DIAMETERS ON DISCHARGE CHARACTERISTICS

Figure 4 shows the electrode surface current density of the $0.2 \mathrm{~mm}$ diameter steel needle is slightly larger than others. When the diameters of the pins are $0.2 \mathrm{~mm}, 0.6 \mathrm{~mm}$, the current density surfaced on the electrode is stable in some areas. Figure 4 shows the smaller the diameter of the electrode is, the greater electrode surface discharge current density is, under the same conditions.

\section{Effect of Distribution Distance of Steel Needle on the Discharge Characteristics}

The distribution distance $\mathrm{D}$ of steel needle is the basic parameter of the multineedle-plate electrode. The size of the multi-pin-plate electrode is $33 \times 33 \mathrm{~mm}$. The numbers of three 
types steel needle are $16 \times 16,8 \times 8,4 \times 4$, respectively. They are distributed evenly on the electrode plate, the distance between the needle ware $2 \mathrm{~mm}, 4 \mathrm{~mm}, 8 \mathrm{~mm}$, respectively. The needle diameter was $1 \mathrm{~mm}$. The length of lower electrode square Copper is $33 \mathrm{~mm}$, the dielectric is covered on the surface of the lower electrode. Change the distribution distance of needle respectively, the distribution of different needle spacing have an effect on the discharge characteristics of the curve. It is shown in Figure 5.

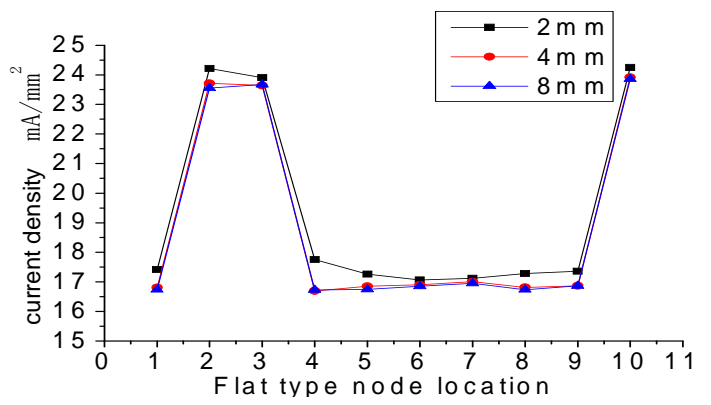

FIGURE V. EFFECT OF THE DISTRIBUTION OF DIFFERENT NEEDLES STEEL SPACING ON DISCHARGE CHARACTERISTICS

Figure 5 shows the three distribution distance of steel needle, the current density covered on the electrodes surface curves have the same trend. When the distribution distance of steel needle are $4 \mathrm{~mm}, 8 \mathrm{~mm}$, the current density covered on the electrode surface of $4 \mathrm{~mm}$ distance is slightly larger than $8 \mathrm{~mm}$. The current density covered on the electrode surface of $2 \mathrm{~mm}$ distance is largest. Compare with Figure 5, under the same conditions, the smaller the distribution distance of steel needle is, the larger current density covered on the electrode surface is.

\section{Effect of Screen Mesh Size on Discharge Characteristics}

The size $\mathrm{H}$ of screen mesh is an important parameter for screen type of electrode structure. In this paper, we select \#40 mesh as standard (the width of mesh is $0.4 \mathrm{~mm}$, the diameter of mesh is $0.2 \mathrm{~mm}$ ), the other types of mesh are set as width of $0.3 \mathrm{~mm}$, diameter of $0.2 \mathrm{~mm}$ and width of $0.6 \mathrm{~mm}$, respectively. The material of mesh electrode is select copper. The distance of the mesh is single changed. The different types of mesh have an effect on the discharge characteristics of the curve. As is shown in Figure 6, the diameters of the pins are $0.3 \mathrm{~mm}, 0.4 \mathrm{~mm}, 0.6 \mathrm{~mm}$, respectively.

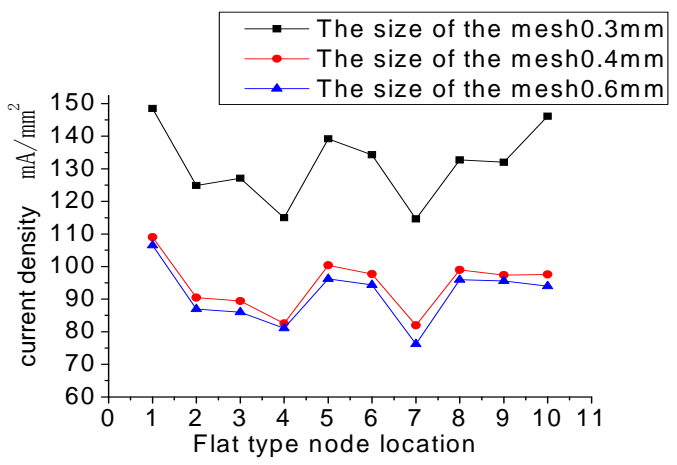

FIGURE VI. EFFECT OF DIFFERENT SCREEN MESH SIZE ON DISCHARGE CHARACTERISTICS
Figure 6 shows the current density covered on the electrodes surface curves have the same trend, for three type of mesh. When the mesh distance is set as $0.3 \mathrm{~mm}, 0.4 \mathrm{~mm}, 0.6 \mathrm{~mm}$, respectively. For mesh distance of $0.3 \mathrm{~mm}$, the current density covered on electrode surface is greater than others. Compare with Figure 6, under the same conditions, the greater the distance of mesh is, the smaller discharge current density of surface layer is. The closer mesh is, the larger current density covered on electrode surface is.

\section{E. Effect of the Structure of Plate-plate, Multineedle-plate, Screen Mesh Electrode on Discharge Characteristics}

The plate-plate, multineedle-plate, screen-mesh optimized electrode structures are compared and the electrode structure parameters suitable for micro-atomization are obtained. As the curve is depicted in the Figure 7. The plate-plate of $1 \mathrm{~mm}$ thickness have an effect on the discharge characteristics of the curve. The different structures have an effect on the discharge characteristics of the curve, such as the needle length of $9 \mathrm{~mm}$, the needle diameter of $0.2 \mathrm{~mm}$, the needle distribution distance of 2mm, the \# 40 mesh screenit . As is shown in Figure 7.

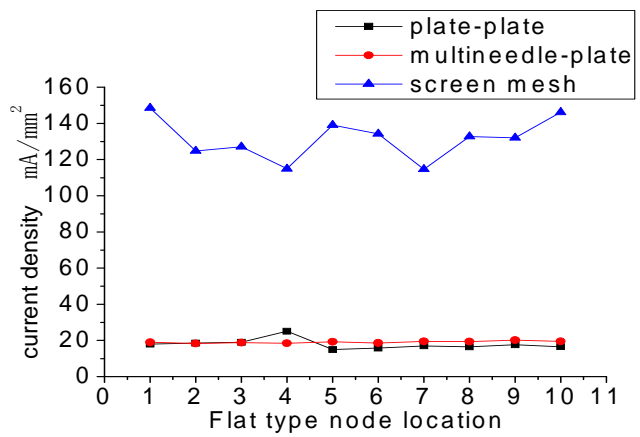

FIGURE VII. EFFECT OF THE STRUCTURE OF PLATE-PLATE, MULTINEEDLE-PLATE, SCREEN MESH ELECTRODE ON DISCHARGE CHARACTERISTICS

Figure 7 shows the current density curve of multi needle plate is almost a straight. The results show that the current density of the multi needle plate electrode is relatively uniform. The current density of the multi needle plate is larger than that of the flat plate electrode surface. The current density covered on the screen type electrode surface is far larger than others. Compare with Figure 7, under the same conditions, the surface current density of the multineedle- plate electrode is more uniform than that of the flat -plate type. The surface current density of the screen mesh electrode is much larger than that of the flat- plate and the multineedle-plate.

\section{CONCLUSION}

In this paper, the Ansys software is used to simulate and analyze the dielectric barrier discharge of flat type suitable for micro-atomizer. The primary research work is to discuss three types of electrode configurations. They have effect on the characteristic of flat-plate micro- atomizer, such as plate-plate, multineedle-plate and screen mesh. Three types of electrode have effect on the characteristic of flat-plate micro-atomizer. The conclusions are as following: 
The simulation results show that the discharge density unit would be easy to obtain by many factors, such as the smaller thickness of plate-plate's electrode, the smaller diameter of steel needle, the smaller distribution of steel needle spacing and the closer mesh. The longer the length of the needle is, the larger current density of the discharge have. Under the same condition, comparison of three types of electrode structure can be kown that the current density covered on the screen mesh's electrode is much larger than the plate-plate and multineedle-plate.

\section{ACKNOWLEDGEMENTS}

This work is supported by National Natural Science Foundation of China $(61463014,61263030)$, Science Research Program of Eduation Bureau of Hubei Province (T201429), Innovation and Entrepreneurship Training Program for College Students in Hubei Province (201510517010).

\section{REFERENCE}

[1] Zhang Jinmao, Liang Jing, Dong Fang. Development of Vapor Generation-Atomic Fluorescence Spectrometer and its Applications in China in last more than thirty years $[\mathrm{J}]$.Chinese Journal of Inorganic Analytical Chemistry,2013,3(4): 1-10.

[2] Wei Lifeng, Luo Yang, Wang Qinghui, etc. Design of Multifunctional Integrated Atomization System [J]. Journal of Shenyang University of Chemical Technology,2015,29(4): 353-357.

[3] Zhou Zhangwen, Liu Zhan, Sun Dong. Diagnosis of Air Density HarassMent [J]. Journal of Jishou University (Natural Science Edition),2011,32(5): 62-64-74.

[4] Wang Xinxin, Li Chengrong. Review of Homogeneous Dielectric Barrier Discharge in Nitrogen at Atmospheric pressure [J]. High Voltage Engineering,2011,37(6): 1405-1415.

[5] Zuo An-you, Yuan Zuo-bin, Weng Zhu-lin, etc. Analysis of the mechanism of the act of plasma on the surface of material [J]. Journal of Hubei University for Nationalities(Natural Sciences Edition),2008,26(2): 173-178.

[6] Hamed Taghvaei, Mahsa Kheirollahivash, Mohammad Ghasemi, et al. Noncatalytic Upgrading of Anisole in an Atmosphere DBD Plasma Reactor: Effect of Carrier Gas Type, Voltage, and Frequency [J]. Energy \&amp; Fuels,2014,28(2): 2535- 2543.

[7] Fahad Rehman, Wamesth S. Abdul Majeed, William B. Zimmerman, et al. Hydrogen Production from Water Vapor Plasmolysis Using DBD-Corona Hybrid Reactor [J]. Energy \&amp; Fuels,2013,27(3): 2748-2761.

[8] Florez, David, Diez, et al. Square-Shape Current-Mode Supply for Parametric Control of the DBD Excilamp Power [J]. IEEE Transactions on Industrial Electronics,2015,62(3): 1451-1460.

[9] Qi Zewu, Zhang Wei, Li Pinglin, etc. Design of DBD high-frequency high-voltage power Supply and its discharging characteristics [J]. High Voltage Engineering,2016,42(3): 807-812.

[10] Zhou Binbin, Fu Hao, Yu Weichuang, et al. Simulation and Study of Flat-plate Dielectric-barrier Discharge Based on Ansys [J]. Journal of Hubei University for Nationalities(Natural Science Edition),2016,34(2): 219-223. 